\title{
On the physical realizability of highly focused electromagnetic field distributions
}

\author{
Rosario Martínez-Herrero, ${ }^{1}$ Ignasi Juvells, ${ }^{2}$ and Artur Carnicer ${ }^{2, *}$ \\ ${ }^{1}$ Universidad Complutense de Madrid, Facultad de Ciencias Físicas, Departamento de Óptica, \\ Ciudad Universitaria, Madrid 28040, Spain \\ ${ }^{2}$ Universitat de Barcelona (UB), Facultat de Física, Departament de Física Aplicada i Òptica, Martí i Franquès 1, \\ Barcelona 28028, Spain \\ *Corresponding author: artur.carnicer@ub.edu
}

Received April 9, 2013; revised May 9, 2013; accepted May 9, 2013; posted May 15, 2013 (Doc. ID 188617); published June 7, 2013

\begin{abstract}
A method to evaluate the physical realizability of an arbitrary three-dimensional vectorial field distribution in the focal area is proposed. A parameter that measures the similarity between the designed (target) field and the physically achievable beam is provided. This analysis is carried out within the framework of the closest electromagnetic field to a given vectorial function, and the procedure is applied to two illustrative cases. (c) 2013 Optical Society of America

OCIS codes: (260.0260) Physical optics; (260.2110) Electromagnetic optics; (260.5430) Polarization.

http://dx.doi.org/10.1364/OL.38.002065
\end{abstract}

Analysis of the electromagnetic field distribution generated in the focal region of an optical focusing system with high numerical aperture (NA) is attracting wide interest because of its multiple applications in electron acceleration, nonlinear optics, and optical tweezers, among others. Since the electric field distribution in the focal plane depends on the complex amplitude and spatial polarization of the beam at the entrance pupil, the incident field has to be conveniently designed [1-11]. In this Letter, we analyze the feasibility of physically implementing an arbitrary three-dimensional vectorial distribution in the focal area. This study is carried out using the framework of the closest electromagnetic field $\mathbf{E}_{\mathbf{F}}$ to a target vectorial function $\mathbf{F}$. Note that this approach allows us to obtain the field $\mathbf{E}_{\mathbf{F}}$, which is a solution of the Maxwell equations that is best fitted in an algebraic sense to $\mathbf{F}[\underline{12}, 13]$. A parameter to evaluate the similarity between the closest field (CF) $\mathbf{E}_{\mathbf{F}}$ and the distribution $\mathbf{F}$ is also introduced.

The electric field distribution at any point in the focal region of a high-NA focusing system is given by the Richards-Wolf integral [1]] :

$$
\begin{aligned}
& \mathbf{E}(r, \phi, z) \\
& =A \int_{0}^{\theta_{0}} \int_{0}^{2 \pi} \mathbf{E}_{\mathbf{0}}(\theta, \varphi) e^{i k \sin \theta r \cos (\phi-\varphi)} e^{-i k \cos \theta z} \sin \theta \mathrm{d} \theta \mathrm{d} \varphi,
\end{aligned}
$$

where $k$ is the wave number, $r$ and $\phi$ are the polar coordinates at the focal plane, $\theta$ and $\varphi$ are the polar and the azimuthal angles, and $A$ is a constant; note that $\theta_{0}=$ $\max \{\theta\}$ is the semi-aperture angle of the aplanatic system (Fig. 1). $\mathbf{E}_{\mathbf{0}}$ is the so-called vectorial angular spectrum, expressed as

$$
\mathbf{E}_{\mathbf{0}}(\theta, \varphi)=P(\theta)\left(f_{1}(\theta, \varphi) \mathbf{e}_{\mathbf{1}}(\varphi)+f_{2}(\theta, \varphi) \mathbf{e}_{2}(\theta, \varphi)\right) .
$$

Here $P(\theta)$ is the so-called apodization function obtained from energy conservation and geometric considerations, and $f_{1}$ and $f_{2}$ are, respectively, the azimuthal and radial components of the incident field, which we assume transverse. The unitary vectors $\mathbf{e}_{1}$ and $\mathbf{e}_{2}$ are given by

$$
\begin{gathered}
\mathbf{e}_{1}(\varphi)=(-\sin \varphi, \cos \varphi, 0) \\
\mathbf{e}_{2}(\theta, \varphi)=(\cos \theta \cos \varphi, \cos \theta \sin \varphi, \sin \theta)
\end{gathered}
$$

Let us assume that $\mathbf{F}(r, \phi, 0)$ is a target distribution to be obtained at the focal plane. The shape of this function is suggested from its potential applications. The problem arises because, in general, $\mathbf{F}$ cannot be written in the form given by Eq. (1). Instead of using $\mathbf{F}$, the CF $\mathbf{E}_{\mathbf{F}}$ associated to $\mathbf{F}$ is used. To clarify what this means, let us suppose that $\mathbf{F}$ is a band-limited function that can be written in terms of its angular plane wave spectrum $\mathbf{F}_{\mathbf{0}}$ :

$$
\mathbf{F}(r, \phi, 0)=A \int_{0}^{\theta_{0}} \int_{0}^{2 \pi} \mathbf{F}_{\mathbf{0}}(\theta, \varphi) e^{i k \sin \theta r \cos (\phi-\varphi)} \sin \theta \mathrm{d} \theta \mathrm{d} \varphi .
$$

Function $\mathbf{F}$ represents the target vectorial field at the focal area. In general, an arbitrary function $\mathbf{F}_{\mathbf{0}}$ does not belong to the subspace $\mathcal{S}$, generated by vectors $\mathbf{e}_{1}$ and $\mathbf{e}_{2}$. From an algebraic point of view, the vector $\mathbf{V}$ belonging to $\mathcal{S}$ and closest to $\mathbf{F}_{\mathbf{0}}$ is given by the projection of $\mathbf{F}_{\mathbf{0}}$ onto the subspace $\mathcal{S}$, namely

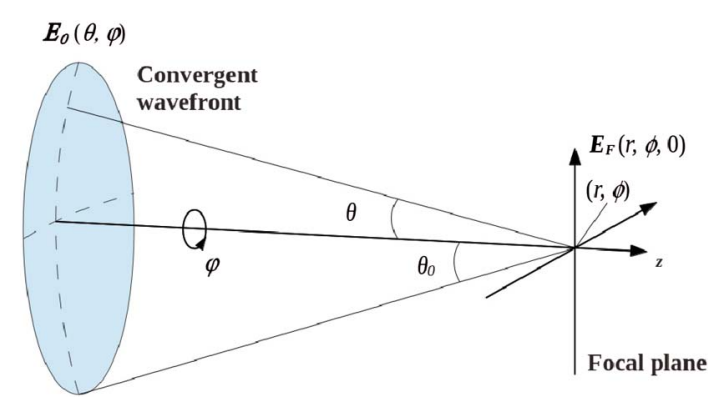

Fig. 1. Notation and geometry of the problem. 


$$
\mathbf{V}=\left(\mathbf{F}_{\mathbf{0}} \cdot \mathbf{e}_{\mathbf{1}}\right) \mathbf{e}_{\mathbf{1}}+\left(\mathbf{F}_{\mathbf{0}} \cdot \mathbf{e}_{2}\right) \mathbf{e}_{\mathbf{2}},
$$

where the dot denotes the inner product. Notice that $\mathbf{V}$ satisfies the following condition at each point $(\theta, \varphi)$ :

$$
\inf \left\{\left|\mathbf{F}_{\mathbf{0}}-\mathbf{G}\right|, G \in S\right\}=\left|\mathbf{F}_{\mathbf{0}}-\mathbf{V}\right|,
$$

where the bars denote the vector modulus (see Fig. 2).

Accordingly, the electric field $\mathbf{E}_{\mathbf{F}}$ closest to $\mathbf{F}$ is defined as

$$
\mathbf{E}_{\mathbf{F}}(r, \phi, 0)=A \int_{0}^{\theta_{0}} \int_{0}^{2 \pi} \mathbf{V}(\theta, \varphi) e^{i k \sin \theta r \cos (\phi-\varphi)} \sin \theta \mathrm{d} \theta \mathrm{d} \varphi .
$$

Obviously, when $\mathbf{F}_{\mathbf{0}}$ belongs to $\mathcal{S}, \mathbf{F}_{\mathbf{0}}=\mathbf{V}$ and therefore $\mathbf{E}_{\mathbf{F}}=\mathbf{F}[\underline{12}, 13]$. To estimate the difference between the CF field $\mathbf{E}_{\mathbf{F}}$ and the target function $\mathbf{F}$, we introduce parameter $\rho$, defined as

$$
\rho=\frac{\int_{0}^{\infty} \int_{0}^{2 \pi}\left|\mathbf{E}_{\mathbf{F}}(r, \phi, 0)\right||\mathbf{F}(r, \phi, 0)| r \mathrm{~d} r \mathrm{~d} \phi}{\sqrt{I_{F} I_{E}}},
$$

where

$$
\begin{gathered}
I_{E}=\int_{0}^{\infty} \int_{0}^{2 \pi}\left|\mathbf{E}_{\mathbf{F}}(r, \phi, 0)\right|^{2} r \mathrm{~d} r \mathrm{~d} \phi, \\
I_{F}=\int_{0}^{\infty} \int_{0}^{2 \pi}|\mathbf{F}(r, \phi, 0)|^{2} r \mathrm{~d} r \mathrm{~d} \phi .
\end{gathered}
$$

Since $\mathbf{V}$ is the projection of $\mathbf{F}_{\mathbf{0}}$ onto subspace $\mathcal{S}, \rho$ provides a measure of the physical realizability of the selected function F. Obviously, $\rho \leq 1$, and by using Parseval's theorem and Hölder's inequality, a lower bound $\rho_{0}$ for $\rho$ can be obtained, namely $\rho_{0} \leq \rho \leq 1$, which is

$$
\rho_{0}=\frac{\left|\int_{0}^{\theta_{0}} \int_{0}^{2 \pi}\left(\mathbf{V} \cdot \mathbf{F}_{\mathbf{0}}\right) \sin \theta \mathrm{d} \theta \mathrm{d} \varphi\right|}{\sqrt{\int_{0}^{\theta_{0}} \int_{0}^{2 \pi}|\mathbf{V}|^{2} \sin \theta \mathrm{d} \theta \mathrm{d} \varphi \int_{0}^{\theta_{0}} \int_{0}^{2 \pi}\left|\mathbf{F}_{\mathbf{0}}\right|^{2} \sin \theta \mathrm{d} \theta \mathrm{d} \varphi}} .
$$

Notice that $\rho$ depends on $\mathbf{F}$ as well as the parameters of the optical system, such as the NA and the pupil function $P(\theta)$. Related to this, a high value for the lower bound

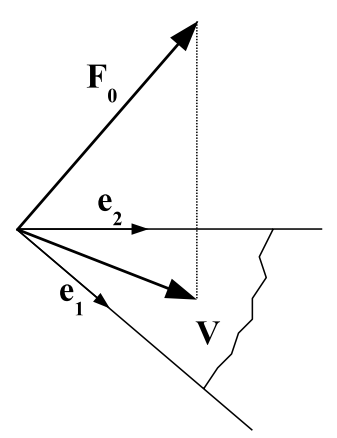

Fig. 2. Projection of $\mathbf{F}_{\mathbf{0}}$ on the subspace $\mathcal{S}$. given in Eq. (10) implies that $\rho$ must be closer to 1 and then $\mathbf{E}_{\mathbf{F}}$ will be best fitted to $\mathbf{F}$.

As an illustrative example, we consider the ideal case of a purely longitudinal vectorial distribution at the focus. It is clear from Eq. (1) that this polarization structure is not physically realizable. Instead, we will consider the CF associated to this pure longitudinal field distribution. In this case, the target wave spectrum takes the form $\mathbf{F}_{\mathbf{0}}=P(\theta) g(\theta, \varphi)(0,0,1), g(\theta, \varphi)$ being an arbitrary function. In this case, vector $\mathbf{V}$ and its corresponding $\mathrm{CF}$ [cf. Eqs. (5) and (7)] are, respectively,

$$
\mathbf{V}(\theta, \varphi)=P(\theta) \sin \theta g(\theta, \varphi) \mathbf{e}_{\mathbf{2}}(\theta, \varphi)
$$

and

$$
\begin{aligned}
\mathbf{E}_{\mathbf{F}}(r, \phi, 0)= & A \int_{0}^{\theta_{0}} \int_{0}^{2 \pi} P(\theta) \sin \theta g(\theta, \varphi) \mathbf{e}_{2}(\theta, \varphi) \\
& \times e^{i k r \sin \theta \cos (\phi-\varphi)} \sin \theta \mathrm{d} \theta \mathrm{d} \varphi .
\end{aligned}
$$

From the equation above it is concluded that an incident beam has to be radially polarized to obtain the $\mathrm{CF}$ associated to a purely longitudinal vectorial distribution at the focal plane. Such a property can be thought of as an analytical support to the well-known result of achieving a significant longitudinal component by focusing radially polarized light. In this case the bound $\rho_{0}$ takes the form

$$
\rho_{0}^{2}=\frac{\int_{0}^{\theta_{0}} \int_{0}^{2 \pi}|P(\theta) g(\theta, \varphi)|^{2} \sin ^{3} \theta \mathrm{d} \theta \mathrm{d} \varphi}{\int_{0}^{\theta_{0}} \int_{0}^{2 \pi}|P(\theta) g(\theta, \varphi)|^{2} \sin \theta \mathrm{d} \theta \mathrm{d} \varphi} .
$$

Additional features for the field $\mathbf{E}_{\mathbf{F}}(r, \phi, 0)$ can be accomplished by choosing suitable functions $P(\theta)$ and $g(\theta, \varphi)$. Taking into account an objective lens obeying the sine condition and assuming rotational symmetry, Eqs. (12) and (13) become

$$
\begin{aligned}
\mathbf{E}_{\mathbf{F}}(r, \phi, 0)= & A \int_{0}^{\theta_{0}} \int_{0}^{2 \pi} \sqrt{\cos \theta} \sin \theta g(\theta) \mathbf{e}_{2}(\theta, \varphi) \\
& \times e^{i k r \sin \theta \cos (\phi-\varphi)} \sin \theta \mathrm{d} \theta \mathrm{d} \varphi .
\end{aligned}
$$

and

$$
\rho_{0}^{2}=\frac{\int_{0}^{\theta_{0}} \int_{0}^{2 \pi} \cos \theta|g(\theta)|^{2} \sin ^{3} \theta \mathrm{d} \theta \mathrm{d} \varphi}{\int_{0}^{\theta_{0}} \int_{0}^{2 \pi} \cos \theta|g(\theta)|^{2} \sin \theta \mathrm{d} \theta \mathrm{d} \varphi} .
$$

It is apparent from Eq. (15) that an apodization of $g(\theta)$ and large semi-aperture angles will maximize the value of $\rho_{0}$; thus $\rho$ will be higher. In fact, from Eq. (11) we get

$$
\lim _{\theta \rightarrow \pi / 2} \mathbf{V}(\theta, \varphi)=\mathbf{F}_{\mathbf{0}}
$$

and therefore $\mathbf{E}_{\mathbf{F}}$ will tend to be a pure longitudinal field. The apodized beam will provide better results because only the contribution of the larger semi-aperture angles $\theta$ is taken into account. In order to show the behavior of $\rho$, two different distributions for $g(\theta)$ have been considered: (i) a Gaussian-Bessel beam and (ii) an apodized Gaussian-Bessel beam: 


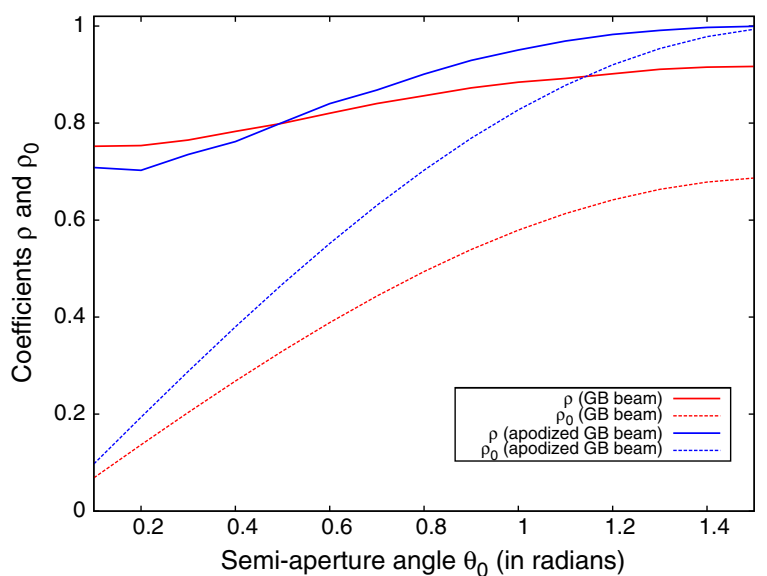

Fig. 3. Coefficients $\rho$ and $\rho_{0}$ versus the semi-aperture angle $\theta_{0}$ (in radians) for a longitudinal-only polarized beam. Two functions $g(\theta)$ are considered: (i) a Gaussian-Bessel beam and (ii) an apodized Gaussian-Bessel beam with $\theta_{\min }=0.95 \theta_{0}$ and $\theta_{\max }=\theta_{0}$. In both cases, $\beta=0.5$.

$$
g(\theta)=\exp \left[-\beta\left(\frac{\sin \theta}{\sin \theta_{0}}\right)^{2}\right] J_{1}\left(2 \beta \frac{\sin \theta}{\sin \theta_{0}}\right),
$$

where $\beta^{-1}$ is the so-called filling factor [15]. The apodized beam is calculated by multiplying $g(\theta)$ by an annulus limited by angles $\theta_{\min }$ and $\theta_{\max }$. Figure 3 shows the behavior of $\rho$ as a function of the semi-aperture angle $\theta_{0}$ the for two beams analyzed. It is apparent from Fig. 3 that higher values of $\rho$ are obtained for large semi-aperture angles and vice versa.

To provide more insight about the behavior of $\rho$ and $\rho_{0}$, we consider now a purely transverse radially polarized distribution at the focus of an objective lens obeying the sine condition. In this case $\mathbf{F}_{\mathbf{0}}$ takes the form $\mathbf{F}_{\mathbf{0}}=\sqrt{\cos \theta} g(\theta)(\cos \varphi, \sin \varphi, 0)$, and therefore

$$
\mathbf{V}(\theta, \varphi)=\sqrt{\cos \theta} \cos \theta g(\theta) \mathbf{e}_{2}(\theta, \varphi) .
$$

As expected, the incident field presents radial polarization. Assuming rotational symmetry, the lower bound for $\rho$ now takes the form

$$
\rho_{0}^{2}=\frac{\int_{0}^{\theta_{0}} \int_{0}^{2 \pi} \cos ^{3} \theta|g(\theta)|^{2} \sin \theta \mathrm{d} \theta \mathrm{d} \varphi}{\int_{0}^{\theta_{0}} \int_{0}^{2 \pi} \cos \theta|g(\theta)|^{2} \sin \theta \mathrm{d} \theta \mathrm{d} \varphi} .
$$

From Eq. (19) and Fig. 4, it can be concluded that apodization is not advisable and better results are obtained for small semi-aperture angles. Notice that now $\lim _{\theta \rightarrow 0} \mathbf{V}(\theta, \varphi)=\mathbf{F}_{\mathbf{0}}$ and $\mathbf{E}_{\mathbf{F}}$ tends to be a purely transverse beam.

In summary, we have shown a procedure to design the input beam in order to obtain a realizable field distribution at the focus of an objective lens. From the two examples considered, we can conclude that physically

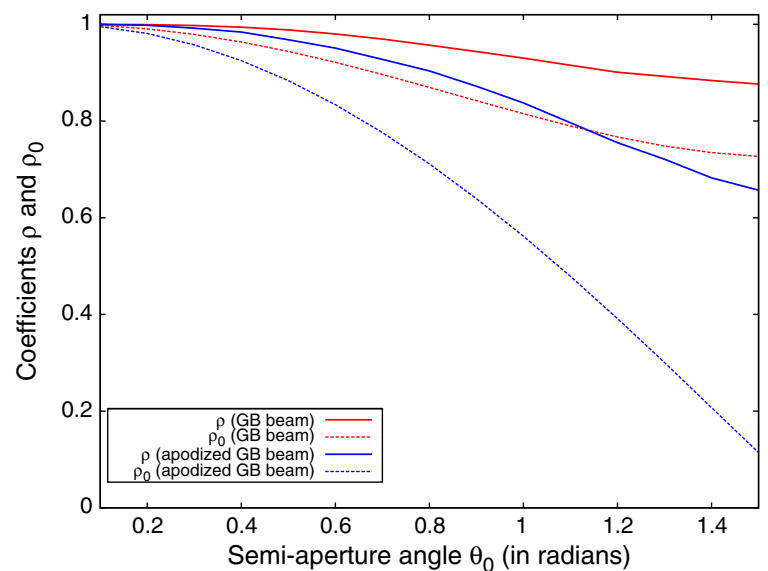

Fig. 4. Coefficients $\rho$ and $\rho_{0}$ versus the semi-aperture angle $\theta_{0}$ (in radians) for a transverse-only radially polarized beam. Functions $g(\theta)$ are the same as in Fig. 3.

realizable beams with a high longitudinal component can be obtained when a radially polarized apodized beam is focused using a high-NA objective lens. On the other hand, apodization is not advisable to generate transverse-only radially polarized beams; better results are obtained for low aperture angles.

This work has been supported by the Ministerio de Ciencia e Innovación of Spain, project FIS2010-17543.

\section{References}

1. R. Dorn, S. Quabis, and G. Leuchs, Phys. Rev. Lett. 91, 233901 (2003).

2. N. Davidson and N. Bokor, Opt. Lett. 29, 1318 (2004).

3. C. J. Sheppard and A. Choudhury, Appl. Opt. 43, 4322 (2004).

4. Y. Kozawa and S. Sato, J. Opt. Soc. Am. A 24, 1793 (2007).

5. H. Wang, L. Shi, B. Lukyanchuk, C. Sheppard, and C. T. Chong, Nat. Photonics 2, 501 (2008).

6. G. M. Lerman and U. Levy, Opt. Express 16, 4567 (2008).

7. K. Kitamura, K. Sakai, and S. Noda, Opt. Express 18, 4518 (2010).

8. X. Hao, C. Kuang, T. Wang, and X. Liu, Opt. Lett. 35, 3928 (2010).

9. H. Chen, Z. Zheng, B.-F. Zhang, J. Ding, and H.-T. Wang, Opt. Lett. 35, 2825 (2010).

10. S. N. Khonina and S. G. Volotovsky, J. Opt. Soc. Am. A 27, $2188(2010)$.

11. F. Kenny, D. Lara, O. Rodríguez-Herrera, and C. Dainty, Opt. Express 20, 14015 (2012).

12. R. Martínez-Herrero, P. M. Mejías, S. Bosch, and A. Carnicer, J. Opt. Soc. Am. A 18, 1678 (2001).

13. R. Martínez-Herrero, P. M. Mejías, and G. Piquero, Characterization of Partially Polarized Light Fields (Springer, 2009), pp. 151-164.

14. B. Richards, E. Wolf, B. Richards, and E. Wolf, Proc. R. Soc. A 253, 358 (1959).

15. L. Novotny and B. Hecht, Principles of Nano-Optics (Cambridge University, 2006), p. 61. 